\title{
Unbiased estimates of cerebrospinal fluid $\beta$-amyloid 1-42 cutoffs in a large memory clinic population
}

\author{
Daniela Bertens ${ }^{1,2}$, Betty M. Tijms ${ }^{1,2}$, Philip Scheltens ${ }^{1,2}$, Charlotte E. Teunissen ${ }^{3}$ and Pieter Jelle Visser ${ }^{1,2,4^{*}}$ (D)
}

\begin{abstract}
Background: We sought to define a cutoff for $\beta$-amyloid 1-42 in cerebrospinal fluid (CSF), a key marker for Alzheimer's disease (AD), with data-driven Gaussian mixture modeling in a memory clinic population.

Methods: We performed a combined cross-sectional and prospective cohort study. We selected 2462 subjects with subjective cognitive decline, mild cognitive impairment, AD-type dementia, and dementia other than AD from the Amsterdam Dementia Cohort. We defined CSF $\beta$-amyloid 1-42 cutoffs by data-driven Gaussian mixture modeling in the total population and in subgroups based on clinical diagnosis, age, and apolipoprotein E (APOE) genotype. We investigated whether abnormal $\beta$-amyloid 1-42 as defined by the data-driven cutoff could better predict progression to AD-type dementia than abnormal $\beta$-amyloid 1-42 defined by a clinical diagnosis-based cutoff using Cox proportional hazards regression.
\end{abstract}

Results: In the total group of patients, we found a cutoff for abnormal CSF $\beta$-amyloid 1-42 of $680 \mathrm{pg} / \mathrm{ml}(95 \% \mathrm{Cl}$ $660-705 \mathrm{pg} / \mathrm{ml}$ ). Similar cutoffs were found within diagnostic and APOE genotype subgroups. The cutoff was higher in elderly subjects than in younger subjects. The data-driven cutoff was higher than our clinical diagnosisbased cutoff and had a better predictive accuracy for progression to AD-type dementia in nondemented subjects (HR 7.6 versus $5.2, p<0.01$ ).

Conclusions: Mixture modeling is a robust method to determine cutoffs for CSF $\beta$-amyloid 1-42. It might better capture biological changes that are related to AD than cutoffs based on clinical diagnosis.

Keywords: Alzheimer's disease, $\mathrm{MCl}$, Cerebrospinal fluid, Diagnosis

\section{Background}

Decreased $\beta$-amyloid 1-42 $\left(\mathrm{A} \beta_{42}\right)$ in cerebrospinal fluid (CSF) is indicative of Alzheimer's disease (AD) pathology and part of research criteria for $\mathrm{AD}[1-4]$. However, there is no universal cutoff value to define abnormal CSF $A \beta_{42}$. This is in part due to the variability of $A \beta_{42}$ measurements across laboratories [5, 6]. In addition, clinical centers have used different methods to define a cutoff [7]. Often, a cutoff value is determined by comparing CSF $A \beta_{42}$ levels of cognitively normal subjects with those of patients with a clinical diagnosis of AD-

\footnotetext{
* Correspondence: pj.visser@vumc.nl

${ }^{1}$ Department of Neurology, Neuroscience Campus Amsterdam, VU University Medical Center, Amsterdam, The Netherlands

${ }^{2}$ Alzheimer Center, Neuroscience Campus Amsterdam, VU University Medical Center, De Boelelaan 1118, 1081, HZ Amsterdam, The Netherlands Full list of author information is available at the end of the article
}

type dementia. However, about $10 \%$ of the subjects with clinical AD-type dementia do not have amyloid pathology [8], and $25 \%$ of subjects with normal cognition can have amyloid pathology [9], which biases the cutoff value. Data-driven Gaussian mixture modeling provides an alternative approach that does not rely on clinical diagnosis [10]. With this approach, CSF $A \beta_{42}$ levels showed a bimodal distribution representing a normal and an abnormal population. However, it is unclear whether this method is influenced by clinical diagnosis and risk factors for AD.

We aimed to define a cutoff for CSF $A \beta_{42}$ with mixture modeling and to investigate whether this cutoff was dependent on clinical diagnosis, age, and apolipoprotein E (APOE) genotype. We compared the diagnostic accuracy and the predictive accuracy for AD-type dementia 
progression of the new cutoff value with our previous clinically based cutoff [11]. We also performed a simulation analysis to determine the minimum sample size needed to reliably estimate the cutoff with mixture modeling.

\section{Methods}

\section{Participants}

We selected 2462 subjects from the Amsterdam Dementia Cohort (ADC) [12] with subjective cognitive decline (SCD; $n=448$ ), mild cognitive impairment (MCI; $n=$ 490), AD dementia $(n=1031)$, and dementia other than AD $(n=493)$ who had CSF measurements available at the time of their first visit at the memory clinic between August 1997 and July 2015. All patients underwent standardized dementia screening at baseline, including physical and neurological examinations, electroencephalograms, magnetic resonance imaging, and laboratory tests. Cognitive screening included the Mini Mental State Examination and, in over $90 \%$ of the subjects, a comprehensive neuropsychological test battery. Diagnoses were made by consensus among a multidisciplinary team that did not have knowledge of the CSF results, and they were based on the following clinical criteria. Subjects were diagnosed with SCD when cognitive complaints were present but criteria for MCI, dementia, or any other neurological or psychiatric disorders were not met and all other examinations were normal [13]. Subjects were diagnosed with MCI according to the established MCI criteria [14]. Subjects with AD-type dementia were diagnosed according to the criteria of the National Institute of Neurological and Communicative Disorders and Stroke-Alzheimer's Disease and Related Disorders Association [4, 15]. Subjects with non-AD-type dementia included subjects with behavioral variants of frontotemporal dementia $(n=204)[16,17]$, dementia with Lewy bodies $(n=113)$ [18], vascular dementia $(n=66)$ [19], corticobasal degeneration or syndrome ( $n=31$ or $n=10$, respectively) [20], progressive supranuclear palsy $(n=44)$ [21], alcoholrelated dementia $(n=3)$, Huntington's disease $(n=3)$, Parkinson's disease $(n=1)$, normal pressure hydrocephalus $(n=3)$, CADASIL (cerebral autosomal dominant arteriopathy with subcortical infarcts and leukoencephalopathy; $n=1)$, Creutzfeldt-Jakob disease $(n=3)$, tauopathy $(n=2)$, and dementia without a known cause $(n=9)$. All subjects gave written informed consent for the use of their clinical and biomarker data for research purposes, and the ethical review board of the VU University Medical Center approved the study.

\section{Follow-up assessment}

Subjects were followed according to clinical needs. The standard follow-up procedure included a 6-month follow-up examination for subjects with dementia and a 12-month follow-up visit for subjects without dementia [12]. Neuropsychological tests were repeated every 12 months. Diagnoses at follow-up were made on the basis of clinical criteria listed above by consensus among a multidisciplinary team.

\section{CSF $A \beta_{1-42}$ analyses}

CSF was collected from $67 \%$ of the subjects in the ADC [12]. Reasons for not collecting CSF were refusal, contraindications, technical failure, or CSF having been collected elsewhere. CSF was obtained by lumbar puncture using a 25 -gauge needle with a syringe into $10-\mathrm{ml}$ polypropylene tubes (Sarstedt, Nümbrecht, Germany). Within $2 \mathrm{~h}$, CSF samples were centrifuged at $1800 \times g$ for 10 minutes at $4{ }^{\circ} \mathrm{C}$. The CSF supernatant was transferred to new polypropylene tubes and stored at $-20{ }^{\circ} \mathrm{C}$ until further analysis (within 2 months). Baseline $A \beta_{1-42}$ was measured using a commercially available enzymelinked immunosorbent assay (Innotest $\beta$-amyloid(1- 42 ; Innogenetics, Ghent, Belgium) on a routine basis as described before [11]. The intra-assay coefficient of variation (mean $\pm \mathrm{SD}$ ) for $A \beta_{1-42}$ was $2.0 \pm 0.5 \%$, calculated by averaging the coefficient of variation of duplicates from five runs randomly selected over 2 years. The inter-assay coefficient of variation (mean \pm SD) was 10.9 $\pm 1.8 \%$, as analyzed in a high and low pool from 13 consecutive pool preparations used in total in 189 runs. The team performing the CSF analysis was unaware of the clinical diagnoses.

\section{Statistical analyses}

Baseline characteristics were compared between diagnostic groups with analysis of variance and KruskalWallis or chi-square tests, where appropriate, using IBM SPSS Statistics version 20.0 software (IBM, Armonk, NY, USA). A difference with a $p$ value less than 0.05 was considered significant. Gaussian mixture modeling was used to define a cutoff for abnormal CSF $A \beta_{42}$ using the $\mathrm{R}$ statistical software program version 3.2.1 mixtools package. First, the number of distributions that best described the data was determined with the $\mathrm{R}$ boot.comp function. Next, we defined a data-driven cutoff as the point where the lines of two fitted normal distributions crossed each other. The main analyses included all subjects. We repeated mixture modeling in subgroups based on diagnosis, age (dichotomized based on the median age of 66.5 years), and APOE $\varepsilon 4$ allele carriership. Bootstrap sampling was used to determine $95 \%$ CIs of the cutoff. Cutoffs were considered to be statistically different between subgroups when their 95\% CIs did not overlap.

New cutoff values were compared with our previously clinically defined cutoff of $550 \mathrm{pg} / \mathrm{ml}$, which was based 
on subjects seen between 2001 and $2007(n=1070)$ [11]. For this comparison, we repeated mixture modeling in a subset of data including subjects from this time period. Using this subset, we further tested differences between the clinical and new cutoff values in discrimination between nondemented subjects with or without AD-type dementia at follow-up. In addition, using Cox proportional hazard models, we compared the association of the old cutoff and new cutoff with time to AD-type dementia progression, including age and sex as covariates. For these analyses, nondemented patients were included when they had at least 6 months of follow-up available. We compared both models with chi-square tests of the $\log$-likelihood ratio. A difference with a $p$ value less than 0.05 was considered significant. Statistical analyses for multivariate Cox regression were performed using $\mathrm{R}$ version 3.2.3 software.

Finally, we studied the minimum number of subjects per clinical population necessary to reliably estimate the cutoff in a data-driven way. To this end, we simulated CSF $A \beta_{42}$ values from a bimodal distribution with mean and SD values as estimated from our dataset. We recalculated the cutoffs and 95\% CIs for sample sizes with varying numbers starting from $n=300$ to 3000 with steps of 100 . The minimum sample size required to obtain a reliable cutoff was determined as the sample size for which 95\% CI lines were larger than the mean cutoff $\pm 10 \%$, which is currently used as a rule-of-thumb indication of acceptable variability in CSF A $\beta_{42}$ levels.

\section{Results}

Baseline characteristics

Table 1 shows the baseline characteristics according to diagnostic group. Briefly, patients with $\mathrm{AD}$ and patients with MCI were older and included a higher percentage of APOE $\varepsilon 4$ allele carriers than the other groups. CSF $A \beta_{42}$ levels were highest for SCD, followed by non-ADtype dementia, $\mathrm{MCI}$, and $\mathrm{AD}$-type dementia. Of the nondemented subjects, over an average follow-up period of 3.2 (SD 2.04) years, 21 (9\%) of the subjects with SCD progressed to $\mathrm{MCI}$ and 13 (5\%) to AD-type dementia, and $146(39 \%)$ of the subjects with MCI progressed to AD-type dementia.

\section{CSF $A \beta_{1-42}$ cutoff based on mixture modeling}

In the total sample and all subgroups, a bimodal distribution best fitted the data (Table 2). In the total sample, this yielded a cutoff of $680 \mathrm{pg} / \mathrm{ml}$ (95\% CI $660-705 \mathrm{pg} /$ ml) (Fig. 1a). With this cutoff, $55 \%$ of our population fell into the abnormal amyloid distribution. Similar cutoffs were found when we repeated mixture modeling within the dementia group $(694 \mathrm{pg} / \mathrm{ml}, 95 \%$ CI $670-721 \mathrm{pg} /$ $\mathrm{ml}$ ), the pooled sample of subjects with SCD and MCI $(664 \mathrm{pg} / \mathrm{ml}$ [95\% CI 621-712 $\mathrm{pg} / \mathrm{ml})$, subjects with SCD $(621 \mathrm{pg} / \mathrm{ml}(95 \%$ CI $526-901 \mathrm{pg} / \mathrm{ml})$ and subjects with MCI $(696 \mathrm{pg} / \mathrm{ml}[95 \%$ CI 654-758 pg/ml) (Fig. 1b-e). A lower cutoff was found for subjects younger than 66.5 years $(645 \mathrm{pg} / \mathrm{ml}[95 \% \mathrm{CI} 617-678 \mathrm{pg} / \mathrm{ml})$ than subjects older than 66.5 years $(723 \mathrm{pg} / \mathrm{ml}$ [95\% CI 691$762 \mathrm{pg} / \mathrm{ml}$ ) (Fig. 1f and g). The cutoff for CSF A $\beta_{42}$ was higher in APOE $\varepsilon 4$ carriers than in noncarriers (resp. $716 \mathrm{pg} / \mathrm{ml}[95 \%$ CI $684-756 \mathrm{pg} / \mathrm{ml} ; 650 \mathrm{pg} / \mathrm{ml}[95 \%$ CI 611-689 pg/ml), but this did not reach statistical significance (Fig. $1 \mathrm{~h}$ and i).

\section{Comparison with previously defined cutoff}

The cutoff of $680 \mathrm{pg} / \mathrm{ml}$ based on mixture modeling was substantially higher than our previously clinically defined cutoff of $550 \mathrm{pg} / \mathrm{ml}$ (95\% CI 531-570 pg/ml) [11]. Repeated mixture modeling in the subset of subjects with

Table 1 Subject characteristics

\begin{tabular}{|c|c|c|c|c|c|}
\hline & $\begin{array}{l}\text { a. All } \\
(n=2462)\end{array}$ & $\begin{array}{l}\text { b. SCD } \\
(n=448)\end{array}$ & $\begin{array}{l}\text { C. MCl } \\
(n=490)\end{array}$ & $\begin{array}{l}\text { d. AD-type dementia } \\
(n=1031)\end{array}$ & $\begin{array}{l}\text { e. Non-AD-type dementia } \\
(n=493)\end{array}$ \\
\hline Age, years & $66.8(7.0)$ & $64.4(6.2)^{b, c, d}$ & $68.2(6.9)^{a, c, c d}$ & $67.3(7.2)^{a, b}$ & $66.9(6.8)^{a, b}$ \\
\hline Female sex, $n(\%)$ & $1049(43)$ & $170(38)^{c}$ & $181(37)^{c}$ & $528(51)^{a, b, d}$ & $170(34)^{c}$ \\
\hline Years of education & $11.1(3.0)$ & $11.9(3.1)^{\mathrm{b}, \mathrm{c}, \mathrm{d}}$ & $11.4(3.2)^{a, c, d}$ & $10.8(2.8)^{a, b}$ & $10.5(2.9)^{a, b}$ \\
\hline MMSE score & $23.6(5.2)$ & $28.3(1.7)^{b, c, d}$ & $26.5(2.5)^{a, c, d}$ & $20.4(5.0)^{\mathrm{a}, \mathrm{b}, \mathrm{d}}$ & $23.0(5.2)^{a, b, c, c,}$ \\
\hline APOE $\varepsilon 4$ allele carriers, $n(\%)$ & $1186(54)$ & $158(35)^{b, c}$ & $242(49)^{a, c, d}$ & $615(60)^{a, b, d}$ & $171(35)^{b, c}$ \\
\hline $\operatorname{CSF} A \beta_{1-42}, \mathrm{pg} / \mathrm{ml}$ & $667(289)$ & $906(277)^{b, c, d}$ & $676(295)^{a, c, d}$ & $504(174)^{a, b, d}$ & $781(278)^{a, b, c}$ \\
\hline CSF tau, pg/ml & $527(401)$ & $317(205)^{b, c, d}$ & $486(313)^{a, c, d}$ & $705(406)^{a, b, d}$ & $394(443)^{a, b, c}$ \\
\hline CSF p-tau, pg/ml & $70(37)$ & $52(25)^{\mathrm{b}, \mathrm{c}}$ & $70(35)^{a, c, d}$ & $88(39)^{a, b, d}$ & $50(25)^{b, c}$ \\
\hline $\begin{array}{l}\text { Outcome at follow-up AD-type/no } \\
\text { AD-type dementia (\% AD-type dementia) }\end{array}$ & - & $13 / 235(5)$ & $146 / 224(61)$ & - & - \\
\hline Average follow-up duration, years & - & $2.93(1.99)$ & $2.41(1.46)$ & - & - \\
\hline
\end{tabular}

Data are mean (SD), unless otherwise specified. Superscript letters indicate that this group shows a statistically significant difference ( $p<.05)$ with other groups as labelled with a,b,c,d or e in the column headers

Abbreviations: $S C D$ Subjective cognitive decline, $M C I$ Mild cognitive impairment, $A D$ Alzheimer's Disease, $A P O E$ Apolipoprotein $E$, CSF Cerebrospinal fluid, $A \beta_{1-42}$ $\beta$-Amyloid 1-42, - Not applicable 
Table 2 Fit statistics from bootstrap to test the null hypothesis of a $K$-component fit versus $(K+1)$-component fit for total sample

\begin{tabular}{|c|c|c|c|c|c|c|}
\hline & \multicolumn{3}{|c|}{ Log-likelihood 1 versus 2 components } & \multicolumn{3}{|c|}{ Log-likelihood 2 versus 3 components } \\
\hline & Observed & Bootstrap (95\% Cl) & $p$ Value & Observed & Bootstrap (95\% Cl) & $p$ Value \\
\hline All subjects & 653.98 & $6.51(1.65-15.69)$ & $<0.001$ & 2.16 & $16.67(0.78-11.15)$ & 0.77 \\
\hline Subjective cognitive decline & 15.64 & $6.12(1.72-13.25)$ & 0.03 & 2.45 & $5.8(0.94-17)$ & 0.74 \\
\hline Mild cognitive impairment & 124.50 & $5.79(1.91-12.63)$ & $<0.001$ & 9.95 & $5.55(0.54-10.82)$ & 0.08 \\
\hline Nondemented & 132.93 & $6.44(1.5-13.1)$ & $<0.001$ & 10.41 & $4.34(0.73-11.3)$ & 0.07 \\
\hline Dementia & 471.81 & $6.01(1.49-13.21)$ & $<0.001$ & 3.61 & $4.34(0.53-9.56)$ & 0.55 \\
\hline Younger than 66.5 years old & 261.16 & $5.86(1.4-12.82)$ & $<0.001$ & 2.02 & $4.64(0.65-9.83)$ & 0.79 \\
\hline Older than 66.5 years old & 397.49 & $6.42(1.82-14.46)$ & $<0.001$ & 9.39 & $4.82(0.68-11.64)$ & 0.11 \\
\hline APOE $\varepsilon 4$ noncarrier & 138.75 & $6(1.77-13.01)$ & $<0.001$ & 4.19 & $6.91(0.79-11.26)$ & 0.41 \\
\hline APOE $\varepsilon 4$ carrier & 367.29 & $5.83(1.89-11.50)$ & $<0.001$ & 10.49 & $7.25(0.6-11.79)$ & 0.07 \\
\hline
\end{tabular}

APOE Apolipoprotein E

CSF analysis in the 2001-2007 period (in which subjects were selected for the clinically based cutoff calculation) also resulted in a higher cutoff $(615 \mathrm{pg} / \mathrm{ml}, 95 \%$ CI $573-$ $673 \mathrm{pg} / \mathrm{ml}$ ) (Fig. 2) than the clinically defined cutoff.

In this subset, using a cutoff value of $615 \mathrm{pg} / \mathrm{ml}, 439$ subjects were classified as having abnormal amyloid, which was a $13 \%$ increase compared with the 390 subjects classified by the clinically defined cutoff. The sensitivity of the cutoff of $615 \mathrm{pg} / \mathrm{ml}$ for AD-type dementia was 0.89 with a specificity of 0.62 . The clinically defined cutoff of $550 \mathrm{pg} / \mathrm{ml}$ resulted in a sensitivity of 0.86 with a specificity of 0.65 . Of the nondemented subjects who later progressed to AD-type dementia, $87 \%$ had CSF $\mathrm{A} \beta_{42}$ levels less than $615 \mathrm{pg} / \mathrm{ml}$, compared with $76 \%$ with CSF $A \beta_{42}$ levels less than $550 \mathrm{pg} / \mathrm{ml}$. For nondemented subjects who did not progress to AD-type dementia, these proportions were $30 \%$ versus $21 \%$, respectively (Table 3 ). Survival analyses showed that both cutoffs were predictive of the time to development of AD-type dementia $(550 \mathrm{pg} / \mathrm{ml}$ cutoff $\mathrm{HR}=5.14,95 \% \mathrm{CI}$ 2.96-8.93; pg/ml; versus $615 \mathrm{pg} / \mathrm{ml}$ cutoff $\mathrm{HR} 7.44,95 \%$ CI 3.74-14.79) (Table 4). The HR for the development of AD-type dementia was significantly greater for the new cutoff of $615 \mathrm{pg} / \mathrm{ml}$ than for the cutoff of $550 \mathrm{pg} / \mathrm{ml}$ $(p<0.001)$.

We further explored why the data-driven cutpoint was somewhat lower in the subset of subjects with CSF analysis in the 2001-2007 period than in the total sample. Additional file 1: Figure S1 shows that the peaks of CSF $A \beta_{42}$ level distributions seem to shift over subsequent years. This could not be explained by a difference in patient population, because the distribution of diagnoses remained comparable over time $\left(X^{2}(45)=61.32\right.$, $p>0.05$ ) (Additional file 1: Table S1). We further explored whether this shift was due to an assay drift, and we repeated all subgroup analyses stratified for the time period when the lumbar puncture was obtained (2001-2007 versus 2008-2015) (see Additional file 1: Table S2 for baseline characteristics). Briefly, the cutpoint in the total group was higher in the 2008-2015 subsample $(697 \mathrm{pg} / \mathrm{ml}$ [675-723 pg/ml]) than in the 2001-2007 subsample (615 pg/ml [573-673 pg/ml]) (Additional file 1: Table S3 and Figure S2 and S3). Subgroup analyses showed that the cutpoint for the dementia group and for APOE $\varepsilon 4$ allele carriers was also higher in the 2008-2015 group than in the 2001-2007 group. Cutpoints for other subgroups did not differ between time periods.

\section{Minimum sample size required}

Additional file 1: Figure S4 shows the mean and SD values for different sample sizes, varying from 300 to 3000 subjects with steps of 100 . The average CSF $A \beta_{42}$ cutoff was $679 \mathrm{pg} / \mathrm{ml}$ and remained similar with increasing sample size, whereas the $95 \%$ CI became narrower. Accepting a maximum deviation from the cutoff of $\pm 10 \%$, a minimum sample size of 800 is required for an acceptable $95 \%$ CI of $637-744 \mathrm{pg} / \mathrm{ml}$. Our determined cutoff of $680 \mathrm{pg} / \mathrm{ml}$ and 95\% CI fell within this range.

\section{Discussion}

Using a data-driven Gaussian mixture modeling approach, we determined a cutoff of $680 \mathrm{pg} / \mathrm{ml}$ for abnormal CSF $A \beta_{42}$ levels. This cutoff was independent of the cognitive stage and APOE genotype. The cutoff was higher in older than in younger subjects. With this new cutoff, a good classification of subjects with underlying $\mathrm{AD}$ pathology was achieved because $88 \%$ of nondemented subjects who later developed AD-type dementia had CSF $A \beta_{42}$ levels below our new cutoff. Our results suggest that mixture modeling is a robust method to determine cutoff values for CSF $A \beta_{42}$.

In the total sample, we found that subjects with $\mathrm{AD}$ related characteristics (dementia, $\mathrm{MCI}$, older age, and APOE $\varepsilon 4$ carriers) fell mainly within the abnormal amyloid distribution and that subjects without AD-related characteristics (SCD, younger age, and APOE $\varepsilon 4$ 


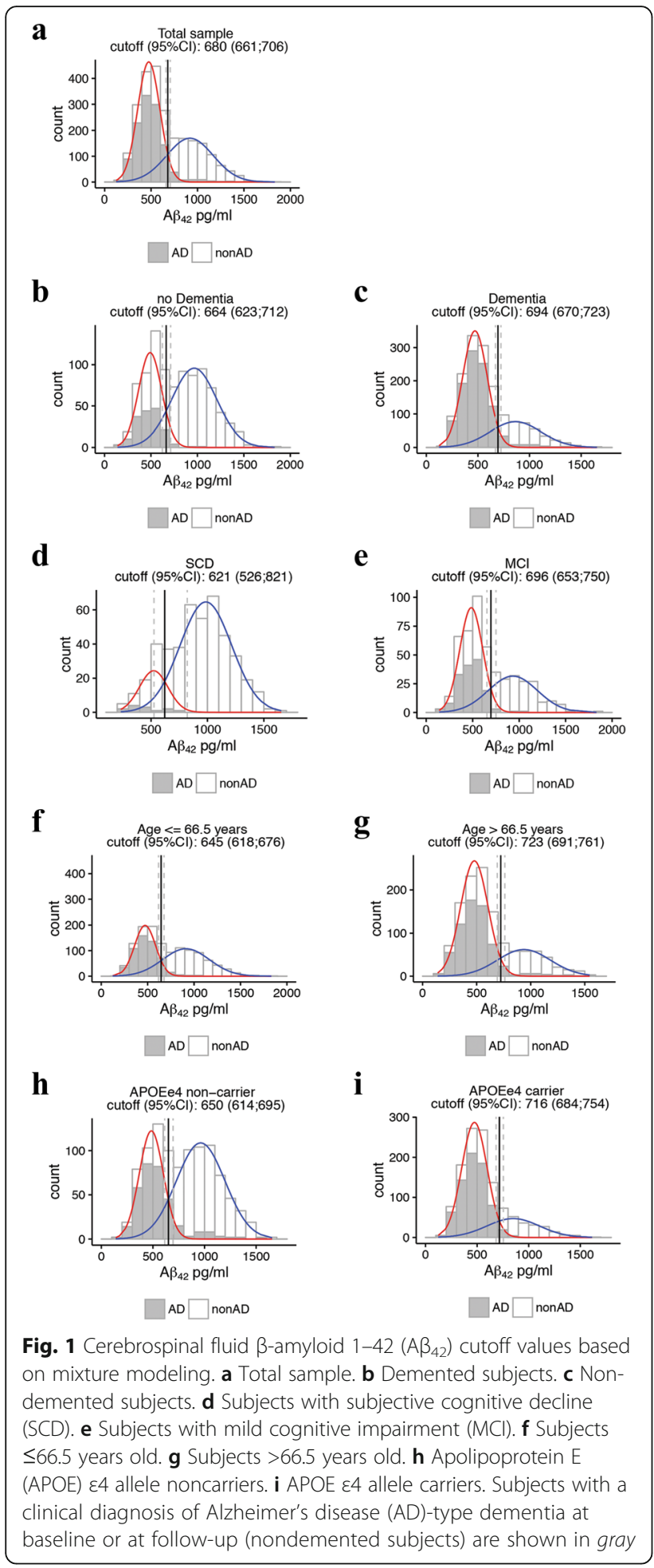

noncarriers) fell mainly within the normal amyloid distribution. This supports the idea that a bimodal distribution of amyloid levels represents normal and abnormal distributions of amyloid. When comparing cutoffs based on age and APOE $\varepsilon 4$ groups, the data-driven cutoff was higher in older than in younger subjects and tended to

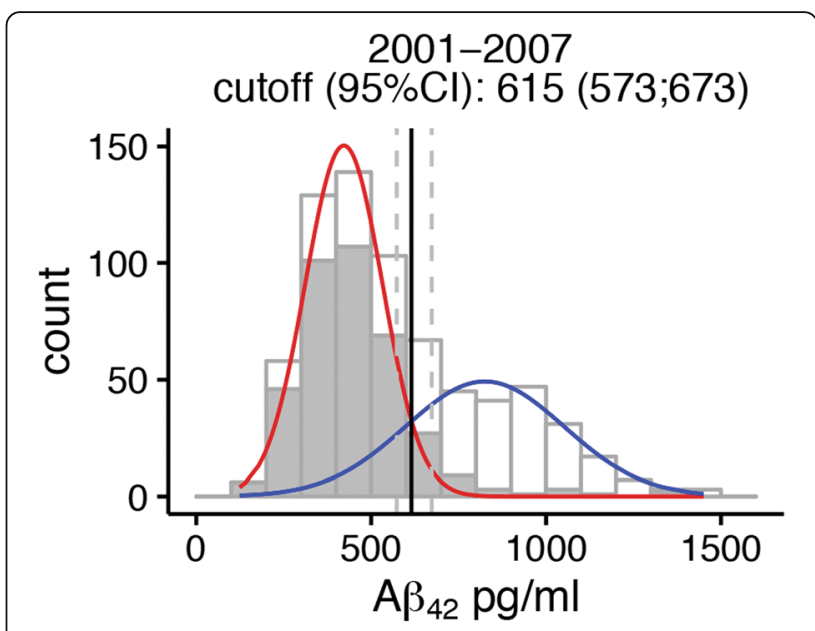

AD nonAD

Fig. 2 Cerebrospinal fluid $\beta$-amyloid 1-42 $\left(A \beta_{42}\right)$ cutoff values based on mixture modeling in subjects seen between 2001 and 2007. Subjects with a clinical diagnosis of Alzheimer's disease (AD)-type dementia at baseline or at follow-up are shown in gray

be higher in APOE \&4 allele carriers than in noncarriers. One explanation for the higher cutoff in these groups is that it reflects a difference in amyloid processing. However, this is unlikely, because a previous study showed that CSF $\mathrm{A} \beta_{42}$ levels were not dependent on APOE $\varepsilon 4$ genotype after correction for $A \beta$ deposition as measured by amyloid positron emission tomography (PET) [22]. Furthermore, researchers in another study found a similar cutoff for CSF $A \beta_{42}$ across age groups [23]. A more likely explanation for the higher cutoff is that, within the old and APOE $\varepsilon 4$ groups, relatively few subjects had normal CSF $A \beta_{42}$ levels. As a consequence, the distribution of values reflecting normal $A \beta_{42}$ levels was relatively wide, which resulted in a shift to a higher cutoff that separated the abnormal and normal CSF $A \beta_{42}$ distribution. Thus, the variability in cutoffs is more likely to result from different sampling frequencies of normal and abnormal $A \beta_{42}$ populations rather than from differences in amyloid processing. This suggests that, in order to determine a cutoff with this data-driven approach, the data need to contain a sufficient sample from both the normal and abnormal populations. For example, for the SCD group, which had few subjects who had abnormal CSF $A \beta_{42}$, the $95 \%$ CI was wide, so the resulting cutpoint should be considered with caution.

The cutoff of $680 \mathrm{pg} / \mathrm{ml}$ defined by Gaussian mixture modeling is higher than our previous clinically defined cutoff of $550 \mathrm{pg} / \mathrm{ml}$. This indicates that clinically based cutoffs may underestimate the presence of abnormal CSF $A \beta_{42}$. Still, the difference in cutoffs may also have resulted from drift in CSF $A \beta_{42}$ levels over time, owing 
Table 3 Number of subjects of subsample between 2001 and 2007 according to diagnosis at baseline, outcome at follow-up, and cerebrospinal fluid $\beta$-amyloid 1-42 cutoff score

\begin{tabular}{llll}
\hline & Total group $(n=688)$ & CSF A $\beta_{42}$ cutoff $<550 \mathrm{pg} / \mathrm{ml}$ & CSF A $\beta_{42}$ Cutoff $<615 \mathrm{pg} / \mathrm{ml}$ \\
\hline AD-type dementia, $n$ (\%) & $288(42)$ & $236(82)$ & $255(89)$ \\
Non-AD-type dementia, $n(\%)$ & $143(21)$ & $49(34)$ & $55(38)$ \\
$\begin{array}{l}\text { SCD with follow-up available } \\
\quad \text { Converted to AD-type dementia, } n(\%)\end{array}$ & $7(7)$ & $5(71)$ & $6(86)$ \\
$\quad$ Not converted to AD-type dementia, $n(\%)$ & $91(93)$ & $16(18)$ & $20(22)$ \\
MCl with follow-up available & & $53(77)$ & $60(87)$ \\
$\quad$ Converted to AD-type dementia, $n(\%)$ & $69(53)$ & $16(27)$ & $26(43)$ \\
$\quad$ Not converted to AD-type dementia, $n(\%)$ & $60(47)$ & &
\end{tabular}

Data are from the subsample of patients seen between 2001 and 2007. For this time period, the cutoff for CSF A $\beta_{42}$ determined with Gaussian mixture modeling was $615 \mathrm{pg} / \mathrm{ml}$

Abbreviations: CSF Cerebrospinal fluid, $A \beta_{42}$ Amyloid- $\beta$ 1-42, SCD Subjective cognitive decline, MCI Mild cognitive impairment, $A D$ Alzheimer's disease

to variability in batches used for the biomarker analysis [6]. To test this possibility, we repeated our analyses based on data obtained in a time period similar to the previously clinically defined cutoff (2001-2007). Indeed, the cutpoint in the 2001-2007 subsample $(615 \mathrm{pg} / \mathrm{ml})$ was lower than that in the 2007-2015 subsample $(697 \mathrm{pg} / \mathrm{ml})$. Nevertheless, the data-driven cutoff derived for the 2007-2015 subsample was still higher than the clinically defined cutoff of $550 \mathrm{pg} / \mathrm{ml}$, suggesting that our higher cutoff value was not due simply to a change in assay performance over time.

Our data-driven cutoff is within the same range as the CSF $A \beta_{42}$ cutoff that shows the best concordance with amyloid PET $(640 \mathrm{pg} / \mathrm{ml})$ in our cohort [24] and in other cohorts $(616-647 \mathrm{pg} / \mathrm{ml})$ in which CSF $\mathrm{A} \beta_{42}$ was assessed with the Innotest assay [25-27]. This similarity between the amyloid PET derived cutoff for CSF A $\beta_{42}$ and our new cutoff suggests that Gaussian mixture modeling is better able than a cutoff based on clinical diagnosis to capture and differentiate subjects from a memory clinic sample in terms of amyloid pathology.

The higher cutoff led to an increased sensitivity to detect subjects with AD-type dementia at baseline and an increased sensitivity to predict future AD-type dementia in subjects with SCD or MCI. This increase in sensitivity could at least in part explain the results of a recent study

Table 4 Cox proportional HRs (95\% Cls) for clinical progression to Alzheimer's disease-type dementia in nondemented subjects

\begin{tabular}{llcl}
\hline CSF A $\beta_{42}$ cutoff & HR $(95 \% \mathrm{Cl})$ & Log-likelihood ratio & $x^{2}$ \\
\hline$<550 \mathrm{pg} / \mathrm{ml}$ & $5.14(2.96-8.93)^{\mathrm{a}}$ & -327.85 & n.a. \\
$<615 \mathrm{pg} / \mathrm{ml}$ & $7.44(3.74-14.79)^{\mathrm{a}}$ & -324.66 & $6.38^{\mathrm{b}}$ \\
\hline
\end{tabular}

Analyses were adjusted for age and sex. Data are from the subsample of nondemented sample with at least 6 months follow-up available seen between 2001 and 2007. The event rate was 33\%, and about $13 \%$ of subjects were lost to follow up per year

Abbreviations: CSF Cerebrospinal fluid, $A \beta_{42} \beta$-Amyloid 1-42, n.a. Not applicable ${ }^{\mathrm{a}} p=0.0001$

${ }^{\mathrm{b}}$ Decrease in $x^{2} p<0.01$ that demonstrated an increased risk for cognitive decline in subjects with low normal CSF $A \beta_{42}$ values, based on a classical clinically defined cutoff [28]. However, subjects with a non-AD type dementia and subjects with SCD and $\mathrm{MCI}$ who did not convert to AD-type dementia also more often had abnormal CSF $A \beta_{42}$ with the new cutoff. Some of these subjects may have low normal scores, but it could also mean that amyloid positivity is typically underestimated in these populations. For example, it is possible that some of the subjects with SCD and MCI might have developed AD-type dementia after the period in which they were followed.

A strength of our study is the availability of a large, clinically well-characterized cohort with longitudinal data. This made it possible to analyze cutoffs for CSF $\mathrm{A} \beta_{42}$ for different subgroups of patients and to assess the ability of the new cutoff to detect future AD-type dementia before cognitive impairment becomes evident. A limitation of the Cox regression analysis was that the time to dementia was an approximation because the event occurred at an unknown time between two visits.

The best gold standard currently available to measure amyloid pathology in vivo is amyloid PET because this correlates strongly with amyloid status determined postmortem [29]. The similarity in cutoffs between our approach and amyloid PET indicates that centers that do not have PET techniques available can improve the accuracy of detecting abnormal amyloid with mixture modeling. A possible limitation of the method, however, is that the sample used for the cutoff definition should contain a sufficient number of subjects with normal and abnormal amyloid. We found that at least 800 subjects are needed to obtain a sufficiently reliable cutoff for CSF $\mathrm{A} \beta_{42}$. This number was calculated under the assumption that, in a memory clinic (as in ours), 55\% of patients have abnormal CSF $\mathrm{A} \beta_{42}$ levels and $45 \%$ have normal levels. However, these distribution parameters might not apply to all memory clinics, because they are dependent 
on the type of patients seen and their age. For example, if $60 \%$ of patients in a population had abnormal CSF $\mathrm{A} \beta_{42}$ levels and $40 \%$ had normal CSF $\mathrm{A} \beta_{42}$ levels, a sample size of at least 450 subjects would be needed to determine a reliable cutoff (data not shown).

\section{Conclusions}

Using a data-driven mixture method, we found a new cutoff for abnormal CSF $A \beta_{42}$ levels that was higher and was better able to predict future AD-type dementia than our clinically determined cutoff. The increase in cutoff for CSF $A \beta_{42}$ has implications for clinical practice because more patients will be labeled as having AD pathology than were so labeled using the old cutoff. This is likely to impact communication with and management of patients [30]. Still, regardless of the cutoff used at this time, the decision whether to communicate abnormal $A \beta_{42}$ values with patients will be challenging because no therapy is yet available. Disclosure of pathological diagnosis will probably depend on the AD stage, the wish of patients to know, and the view of the clinician regarding this topic [31].

\section{Additional file}

Additional file 1: Comparison with previously defined cutoff. (PDF $2355 \mathrm{~kb}$ )

\section{Abbreviations}

AD: Alzheimer's disease; ADC: Amsterdam Dementia Cohort; APOE: Apolipoprotein E; A $\beta$ : $\beta$-Amyloid; CSF: Cerebrospinal fluid; $\mathrm{MCl}$ : Mild cognitive impairment; MMSE: Mini Mental State Examination; PET: Positron emission tomography; p-tau: Phosphorylated tau; SCD: Subjective cognitive decline

\section{Acknowledgements}

Not applicable.

\section{Funding}

This study was supported by the Joint Programme Initiative on Neurodegenerative Disorders and ZonMw (BIOMARKAPD project) and by the Innovative Medicines Initiative Joint Undertaking under grant agreement 115372 (European Medical Information Framework), resources of which are composed of financial contributions from the European Union's Seventh Framework Programme (FP7/2007-2013) and European Federation of Pharmaceutical Industries and Associations companies' in-kind contributions. The funders were not involved in the analysis or reporting of the data.

\section{Availability of data and material}

The datasets analyzed during the present study are available from the corresponding author on reasonable request

\section{Authors' contributions}

DB performed the statistical analysis, interpreted the data, and drafted the manuscript. BMT participated in the design of the study, performed the statistical analysis, and helped to revise the manuscript. PS participated in the design of the study and helped to revise the manuscript. CET participated in the design of the study, supervised the CSF analysis, interpreted the data, and helped to revise the manuscript. PJV conceived of the study, participated in its design and coordination, and helped to draft the manuscript. All authors read and approved the final manuscript and are accountable for all aspects of the work.

\section{Competing interests}

The authors declare that they have no competing interests.

\section{Consent for publication}

Not applicable.

Ethics approval and consent to participate

All subjects gave written informed consent for the use of their clinical and biomarker data for research purposes, and the VU University Medical Center ethics committee approved the study.

\section{Author details}

${ }^{1}$ Department of Neurology, Neuroscience Campus Amsterdam, VU University Medical Center, Amsterdam, The Netherlands. ${ }^{2}$ Alzheimer Center, Neuroscience Campus Amsterdam, VU University Medical Center, De Boelelaan 1118, 1081, HZ Amsterdam, The Netherlands. ${ }^{3}$ Department of Clinical Chemistry, Neurochemistry Lab and Biobank, Neuroscience Campus Amsterdam, VU University Medical Center, Amsterdam, The Netherlands. ${ }^{4}$ Alzheimer Center, School for Mental Health and Neuroscience (MHeNS), University Medical Centre Maastricht, Maastricht, The Netherlands.

Received: 17 August 2016 Accepted: 28 December 2016

Published online: 14 February 2017

\section{References}

1. Dubois B, Feldman HH, Jacova C, Hampel H, Molinuevo JL, Blennow K, et al. Position Paper Advancing research diagnostic criteria for Alzheimer's disease: the IWG-2 criteria. Lancet Neurol. 2014;13(6):614-29.

2. Sperling RA, Aisen PS, Beckett LA, Bennett DA, Craft S, Fagan AM, et al. Toward defining the preclinical stages of Alzheimer's disease: recommendations from the National Institute on Aging-Alzheimer's Association workgroups on diagnostic guidelines for Alzheimer's disease. Alzheimers Dement. 2011;7(3):280-92.

3. Albert MS, DeKosky ST, Dickson D, Dubois B, Feldman HH, Fox NC, et al. The diagnosis of mild cognitive impairment due to Alzheimer's disease: recommendations from the National Institute on Aging-Alzheimer's Association workgroups on diagnostic guidelines for Alzheimer's disease. Alzheimers Dement. 2011;7(3):270-9.

4. McKhann GM, Knopman DS, Chertkow H, Hyman BT, Jack Jr CR, Kawas CH, et al. The diagnosis of dementia due to Alzheimer's disease: recommendations from the National Institute on Aging-Alzheimer's Association workgroups on diagnostic guidelines for Alzheimer's disease. Alzheimers Dement. 2011;7(3):263-9.

5. Mattsson N, Andreasson U, Persson S, Carrillo MC, Collins S, Chalbot S, et al. CSF biomarker variability in the Alzheimer's Association quality control program. Alzheimer's Dement. 2013;9(3):251-61.

6. Vos SJB, Visser PJ, Verhey F, Aalten P, Knol D, Ramakers I, et al. Variability of CSF Alzheimer's disease biomarkers: implications for clinical practice. PLOS One. 2014;9(6):e100784.

7. Bartlett JW, Frost C, Mattsson N, Skillbäck T, Blennow K, Zetterberg H, et al. Determining cut-points for Alzheimer's disease biomarkers: statistical issues, methods and challenges. Biomark Med. 2012;6(4):391-400.

8. Ossenkoppele R, Jansen WJ, Rabinovici GD, Knol DL, van der Flier WM, van Berckel BN, et al. Prevalence of amyloid PET positivity in dementia syndromes: a meta-analysis. JAMA. 2015;313(19):1939-49.

9. Jansen WJ, Ossenkoppele R, Knol DL, Tijms BM, Scheltens P, Verhey FR, et al. Prevalence of cerebral amyloid pathology in persons without dementia: a meta-analysis. JAMA. 2015;313(19):1924-38.

10. De Meyer G, Shapiro F, Vanderstichele H, Vanmechelen E, Engelborghs S, De Deyn PP, et al. Diagnosis-independent Alzheimer disease biomarker signature in cognitively normal elderly people. Arch Neurol. 2010;67(8):949-56.

11. Mulder C, Verwey NA, van der Flier WM, Bouwman FH, Kok A, van Elk EJ, et al. Amyloid- $\beta(1-42)$, total tau, and phosphorylated tau as cerebrospinal fluid biomarkers for the diagnosis of Alzheimer disease. Clin Chem. 2010;56(2):248-53.

12. van der Flier WM, Pijnenburg YAL, Prins N, Lemstra AW, Bouwman FH, Teunissen CE, et al. Optimizing patient care and research: the Amsterdam Dementia Cohort. J Alzheimers Dis. 2014;41(1):313-27.

13. Jessen F, Amariglio RE, van Boxtel M, Breteler M, Ceccaldi M, Chételat G, et al. A conceptual framework for research on subjective cognitive decline in preclinical Alzheimer's disease. Alzheimers Dement. 2014;10(6):844-52. 
14. Petersen RC, Stevens JC, Ganguli M, Tangalos EG, Cummings JL, DeKosky ST. Practice parameter: early detection of dementia: mild cognitive impairment (an evidence-based review). Report of the Quality Standards Subcommittee of the American Academy of Neurology. Neurology. 2001;56(9):1133-42.

15. McKhann G, Drachman D, Folstein M, Katzman R, Price D, Stadlan EM. Clinical diagnosis of Alzheimer's disease: report of the NINCDS-ADRDA Work Group under the auspices of Department of Health and Human Services Task Force on Alzheimer's Disease. Neurology. 1984;34(7):939-44.

16. Neary D, Snowden JS, Gustafson L, Passant U, Stuss D, Black S, et al. Frontotemporal lobar degeneration: a consensus on clinical diagnostic criteria. Neurology. 1998;51(6):1546-54.

17. Rascovsky K, Hodges JR, Knopman D, Mendez MF, Kramer JH, Neuhaus J, et al. Sensitivity of revised diagnostic criteria for the behavioural variant of frontotemporal dementia. Brain. 2011;134(Pt 9):2456-77.

18. McKeith IG, Dickson DW, Lowe J, Emre M, O'Brien JT, Feldman H, et al. Diagnosis and management of dementia with Lewy bodies: third report of the DLB Consortium. Neurology. 2005;65(12):1863-72.

19. Román GC, Tatemichi TK, Erkinjuntti T, Cummings JL, Masdeu JC, Garcia JH, et al. Vascular dementia: diagnostic criteria for research studies. Report of the NINDS-AIREN International Workshop. Neurology. 1993;43(2):250-60.

20. Boeve BF, Lang AE, Litvan I. Corticobasal degeneration and its relationship to progressive supranuclear palsy and frontotemporal dementia. Ann Neurol. 2003;54 Suppl 5:S15-9.

21. Litvan I, Agid Y, Calne D, Campbell G, Dubois B, Duvoisin RC, et al. Clinical research criteria for the diagnosis of progressive supranuclear palsy (SteeleRichardson-Olszewski syndrome): report of the NINDS-SPSP international workshop. Neurology. 1996;47(1):1-9.

22. Lautner R, Palmqvist S, Mattsson N, Andreasson U, Wallin A, Pålsson E, et al. Apolipoprotein E genotype and the diagnostic accuracy of cerebrospinal fluid biomarkers for Alzheimer disease. JAMA Psychiatry. 2014;71(10):1183-91.

23. Mattsson N, Rosén E, Hansson O, Andreasen N, Parnetti L, Jonsson M, et al. Age and diagnostic performance of Alzheimer disease CSF biomarkers. Neurology. 2012;78(7):468-76

24. Zwan M, van Harten A, Ossenkoppele R, Bouwman F, Teunissen C, Adriaanse $\mathrm{S}$, et al. Concordance between cerebrospinal fluid biomarkers and $\left[{ }^{11} \mathrm{C}\right]$ PIB PET in a memory clinic cohort. J Alzheimers Dis. 2014;41(3):801-7.

25. Palmqvist S, Zetterberg H, Blennow K, Vestsberg S, Andreasson U, Brooks DJ, et al. Accuracy of brain amyloid detection in clinical practice using cerebrospinal fluid $\beta$-amyloid 42: a cross-validation study against amyloid positron emission tomography. JAMA Neurol. 2014;71(10):1282-9.

26. Zwan MD, Rinne JO, Hasselbalch SG, Nordberg A, Lleó A, Herukka SK, et al. Use of amyloid-PET to determine cutpoints for CSF markers: a multicenter study. Neurology. 2016;86(1):50-8.

27. Weston PSJ, Paterson RW, Modat M, Burgos N, Cardoso MJ, Magdalinou N, et al. Using florbetapir positron emission tomography to explore cerebrospinal fluid cut points and gray zones in small sample sizes. Alzheimers Dement (Amst). 2015;1(4):440-6.

28. Mattsson N, Insel PS, Donohue M, Jagust W, Sperling R, Aisen P, et al. Predicting reduction of cerebrospinal fluid $\beta$-amyloid 42 in cognitively healthy controls. JAMA Neurol. 2015;72(5):554-60.

29. Clark CM, Pontecorvo MJ, Beach TG, Bedell BJ, Coleman RE, Doraiswamy PM, et al. Cerebral PET with florbetapir compared with neuropathology at autopsy for detection of neuritic amyloid- $\beta$ plaques: a prospective cohort study. Lancet Neurol. 2012;11(8):669-78.

30. Bocchetta M, Galluzzi S, Kehoe PG, Aguera E, Bernabei R, Bullock R, et al. The use of biomarkers for the etiologic diagnosis of $\mathrm{MCl}$ in Europe: an EADC survey. Alzheimers Dement. 2015;11(2):195-206.e1.

31. Visser PJ, Wolf H, Frisoni G, Gertz HJ. Disclosure of Alzheimer's disease biomarker status in subjects with mild cognitive impairment. Biomark Med. 2012;6(4):365-8

\section{Submit your next manuscript to BioMed Central and we will help you at every step:}

- We accept pre-submission inquiries

- Our selector tool helps you to find the most relevant journal

- We provide round the clock customer support

- Convenient online submission

- Thorough peer review

- Inclusion in PubMed and all major indexing services

- Maximum visibility for your research

Submit your manuscript at www.biomedcentral.com/submit
O) Biomed Central 